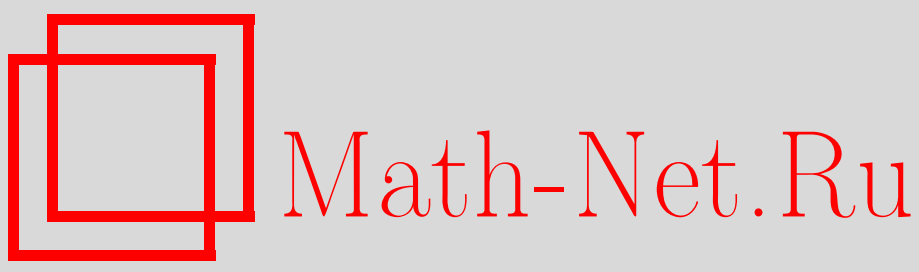

В. Р. Фаталов, Метод Лапласа для малых уклонений гауссовских процессов типа винеровского, Матем. сб., 2005, том 196, номер 4, 135-160

DOI: https://doi.org/10.4213/sm1289

Использование Общероссийского математического портала Math-Net.Ru подразумевает, что вы прочитали и согласны с пользовательским соглашением

http: //www. mathnet.ru/rus/agreement

Параметры загрузки:

IP: 3.85 .5 .30

26 апреля 2023 г., 14:02:03 
УДК 519.2

\author{
В.Р. Фаталов
}

\title{
Метод Лапласа для малых уклонений гауссовских процессов типа винеровского
}

В статье доказаны результаты о точных асимптотиках вероятностей

$$
\mathrm{P}\left\{\int_{0}^{1}|\xi(t)|^{p} d t \leqslant \varepsilon^{p}\right\}, \quad \varepsilon \rightarrow 0
$$

при $p>0$ для двух гауссовских процессов $\xi(t)$ - винеровского процесса и броуновского моста.

Метод исследования - метод Лапласа в банаховых пространствах, подход к вероятностям малых уклонений на основе теории болшших уклонений для времен пребьвания. Вычисления проведены для случаев $p=1$ и $p=2$ в результате решения экстремальной задачи для функционала действия и исследования соответствующих уравнений Шрёдингера.

Библиографияя: 39 названий.

\section{$\S 1$. Введение и формулировка основных результатов}

Изучение малых уклонений гауссовских мер - сравнительно новое направление в области предельных теорем теории вероятностей [1]-[5]. Задача о малых уклонениях связана с исследованием асимптотического поведения вероятности

$$
\mu\left(\varepsilon D_{0}\right), \quad \varepsilon \rightarrow 0,
$$

где $D_{0}$ - некоторая область банахова пространства $(B,\|\cdot\|)$ (например, $D_{0}=$ $\{x \in B:\|x\| \leqslant 1\}), \mu$ - гауссовская мера, заданная на $B$.

В отличие от теории больших уклонений вероятностных мер [6], [7] теория малых уклонений в настоящее время находится в стадии становления. В значительной степени это связано с большей сложностью задачи о малых уклонениях, которая имеет ярко выраженную бесконечномерную природу. Дело в том, что асимптотика вероятности (1.1) для конечномерных пространств является степенной, а в случае бесконечномерных пространств - экспоненциальной. $\mathrm{K}$ настоящему времени разными, довольно изощренными способами вычислены логарифмические асимптотики вероятности (1.1) для разных норм (см. обзор [3] и работу [5]). Намного меньше известно о точных асимптотиках. Хорошо изучен случай гильбертова пространства [1], [2], [8], а также норма супремума для распределений винеровского процесса и броуновского моста.

В настоящей работе мы изучим тот случай малых уклонений, когда мера $\mu=\mu_{w}$ - распределение стандартного винеровского процесса $w(t), t \geqslant 0, w(0)=0$, или

Работа вьполнена при поддержке Российского фонда фундаментальных исследований (грант № 04-01-00700).

(C) В. Р. ФАТАЛОв 2005 
$\mu=\mu_{w_{0}}-$ распределение броуновского моста $w_{0}(t)=\{w(t) \mid w(1)=0\}, \mathrm{a}$ множество $D_{0}$ имеет вид

$$
D=\{x: G(x) \leqslant 1\}
$$

где

$$
G(x)=\|x\|_{p}^{p}=\int_{0}^{1}|x(t)|^{p} d t, \quad p>0,
$$

при $p \geqslant 1$ функционал $G(x)$ представляет собой $p$-ю степень $L^{p}$-нормы.

В статье [4; лемма 2.3] впервые строго доказан (на основе теории гауссовских неравенств) результат о логарифмической асимптотике вероятностей

$$
\mu(\varepsilon D) \equiv \mu\left\{x:\|x\|_{p} \leqslant \varepsilon\right\}, \quad \varepsilon \rightarrow 0, \quad p \geqslant 1,
$$

для $\mu=\mu_{w}$ и $\mu=\mu_{w_{0}}$ (см. также леммы 3 и 4 ниже), хотя сам этот результат в неявной форме содержался уже в работе [9; $(4.5)]$. Отметим также, что некоторые выводы, сделанные в статье [10], нуждаются в уточнении.

Мы покажем, что вычисление точной асимптотики вероятности (1.4) удобно проводить на основе теории больших уклонений для времен пребывания рассматриваемого процесса, привлекая метод Лапласа (см. [6; §5.1], [11]). Напомним, что в работе [11] была вычислена точная асимптотика при $T \rightarrow \infty$ интеграла $\mathrm{E} \exp \left\{T \Psi\left(L_{T}(w, \cdot)\right)\right\}$, где $\mathrm{E}$ - оператор математического ожидания, $L_{t}(w, \cdot)$ - функционал времени пребывания винеровского процесса (см. определение (2.1) ниже) и $\Psi$ - гладкий нелинейный функционал, заданный на пространстве вероятностных мер на $\mathbb{R}$ (ср. с теоремой 4 ниже). Брать в качестве $\Psi$ индикаторную функиию нельзя.

Известно, что в теории больших уклонений переход от точных асимптотик интегралов, взятых по всему пространству, к точным асимптотикам распределений (т.е. интегралов по множествам) является чрезвычайно сложньм и требует особых методов исследования. На это обстоятельство указано в работах [12; I, c. 169], [11; с. 535]. При исследовании асимптотик интегралов по множествам основную роль начинает играть центральная предельная теорема и связанный с ней тонкий гауссовский анализ.

В случае гауссовских мер и распределений сумм независимых банаховозначных случайных элементов упомянутый переход был выполнен автором в статьях [13], [14], причем уже в гауссовском случае в ответе для точной асимптотики распределений появляется константа необычного вида.

В настоящей работе, фактически, осуществлен указанный нетривиальньй переход в случае распределений времен пребывания (см. предложение 1 ниже).

Заметим, что развиваемый ниже метод носит общий характер, он применим в случае иных интегральных функционалов $G$, а также для иных марковских (а возможно, и гауссовских) процессов.

Перейдем к точной формулировке полученных результатов.

Пусть фиксировано число $p>0$. Введем основное гильбертово пространство функций, заданных на $\mathbb{R}$,

$$
L^{2}:=\{v(x), x \in \mathbb{R}: N(v)<\infty\},
$$

где

$$
N(v) \equiv\|v\|^{2}:=\int_{-\infty}^{\infty} v^{2}(x) d x
$$


скалярное произведение в $L^{2}$ обозначим через $\langle\cdot, \cdot\rangle$ :

$$
\langle u, v\rangle:=\int_{-\infty}^{\infty} u(x) v(x) d x, \quad u, v \in L^{2} .
$$

Пусть $\varkappa_{0}>0$ - минимальное собственное число самосопряженного оператора Шрёдингера $\mathscr{B}: L^{2} \rightarrow L^{2}$, имеюшего вид

$$
\left\{\begin{array}{l}
\mathscr{B} y(t)=-\frac{1}{2} y^{\prime \prime}(t)+|t|^{p} y(t), \quad t \in \mathbb{R} \\
y(-\infty)=y(\infty)=0
\end{array}\right.
$$

и $y_{0}(t) \geqslant 0$ - соответствующая нормированная собственная функция, $\left\|y_{0}\right\|=1$ (см. [15; ч. 2, (92)], [16; гл. 4, §7, п. 4; гл. 9, § 2, п. 2]).

Введем важные параметры

$$
\lambda_{0}:=\left(\frac{2 \varkappa_{0}}{2+p}\right)^{(2+p) / p}, \quad \beta_{0}:=\frac{2+p}{2} \lambda_{0}=\left(\frac{2}{2+p}\right)^{2 / p} \varkappa_{0}^{(2+p) / p}
$$

и функции

$$
\begin{aligned}
v_{0}(x) & :=\lambda_{0}^{1 /(2(2+p))} y_{0}\left(\lambda_{0}^{1 /(2+p)} x\right), & & x \in \mathbb{R} \\
V(x) & :=\beta_{0}-\lambda_{0}|x|^{p}, & & x \in \mathbb{R} .
\end{aligned}
$$

Ниже будет показано (см. (3.14)), что

$$
\int_{-\infty}^{\infty} v_{0}^{2}(x) d x=1, \quad \int_{-\infty}^{\infty}|x|^{p} v_{0}^{2}(x) d x=1
$$

(здесь первое равенство легко следует из (1.9) и того, что $\left\|y_{0}\right\|=1$ ).

Мы будем обозначать также символом $V$ оператор умножения, который определен на множестве $\mathscr{D}_{V}$ функций $h \in L^{2}$ таких, что $V h \in L^{2}$. Напомним, что производящий оператор $\mathscr{A}$ винеровского процесса определен на множестве

$$
\begin{aligned}
& \mathscr{D}_{\mathscr{A}}:=\{\text { множество ограниченных на } \mathbb{R} \text { функций } h \text { таких, что } \\
& \left.h, h^{\prime} \text { абсолютно непрерьвны, функция } h^{\prime \prime} \text { ограничена }\right\}
\end{aligned}
$$

и действует по правилу

$$
(\mathscr{A} h)(x)=\frac{1}{2} h^{\prime \prime}(x), \quad h \in \mathscr{D} \mathscr{A}
$$

(см., например, [17; теорема 2.14], [18; гл. 3, п. 22]). Введем множество $\widetilde{\mathscr{D}}_{\mathscr{A}}:=\{h \in$ $\left.\mathscr{D}_{\mathscr{A}} \cap L^{2}: h^{\prime \prime} \in L^{2}\right\}$. На всюду плотном в $L^{2}$ множестве $\mathscr{D}:=\mathscr{D}_{V} \cap \widetilde{\mathscr{D}}_{\mathscr{A}}$ определен оператор $\mathscr{A}+V: L^{2} \rightarrow L^{2}$. Ниже будет показано (см. (3.11)), что ядро $E_{0}$ оператора $\mathscr{A}+V$ представляет собой одномерное подпространство пространства $L^{2}$ :

$$
E_{0}:=\operatorname{Ker}(\mathscr{A}+V)=\left\{c_{0} v_{0}(x), c_{0} \in \mathbb{R}\right\}
$$


Имеет место разложение гильбертова пространства $L^{2}$ в прямую сумму

$$
L^{2}=E_{0} \oplus E_{1}
$$

где

$$
E_{1}:=E_{0}^{\perp}=\left\{v \in L^{2}: \int_{-\infty}^{\infty} v(x) v_{0}(x) d x=0\right\}
$$

- ортогональное дополнение подпространства $E_{0}$ (см. [16; гл. $4, \S 1$, п. 3]). Поскольку оператор $\mathscr{A}+V$ является самосопряженным, то его сужение на подпространство $E_{1}$ (которое вновь обозначается через $\mathscr{A}+V$ ) отображает $E_{1}$ в $E_{1}$ взаимно однозначно (ср. [19; гл. 9, п. 4.5]). Поэтому определен обратный оператор $(\mathscr{A}+V)^{-1} y$, $y \in E_{1}$.

Положим

$$
\psi(x):=|x|^{p}-1, \quad x \in \mathbb{R},
$$

тогда в силу (1.11) $v_{0} \psi \in E_{1}$. Обозначим

$$
\sigma^{2}:=-2 \lambda_{0}^{2} \int_{-\infty}^{\infty}(\mathscr{A}+V)^{-1}\left[v_{0} \psi\right](x)|x|^{p} v_{0}(x) d x
$$

где согласно вышеизложенному обратньй элемент $(\mathscr{A}+V)^{-1}\left[v_{0} \psi\right](x)$ таков, что

$$
\int_{-\infty}^{\infty}(\mathscr{A}+V)^{-1}\left[v_{0} \psi\right](x) v_{0}(x) d x=0 .
$$

Обозначим

$$
b_{p}:=\beta_{0}-\lambda_{0}=p 2^{2 / p}\left(\frac{\varkappa_{0}}{2+p}\right)^{(2+p) / p} .
$$

ТЕорема 1 (точная асимптотика малых уклонений для $L^{p}$-функционалов). Для $p>0$ при $\varepsilon \rightarrow 0$ имеют место соотношения

$$
\begin{aligned}
\mu_{w}\left\{x:\|x\|_{p} \leqslant \varepsilon\right\} & \equiv \mathrm{P}\left\{\int_{0}^{1}|w(t)|^{p} d t \leqslant \varepsilon^{p}\right\} \\
& =\varepsilon \exp \left\{-\frac{b_{p}}{\varepsilon^{2}}\right\} v_{0}(0) \int_{-\infty}^{\infty} v_{0}(x) d x \frac{1}{\sqrt{2 \pi} \sigma}(1+o(1)), \\
\mu_{w_{0}}\left\{x:\|x\|_{p} \leqslant \varepsilon\right\} & \equiv \mathrm{P}\left\{\int_{0}^{1}\left|w_{0}(t)\right|^{p} d t \leqslant \varepsilon^{p}\right\} \\
& =\exp \left\{-\frac{b_{p}}{\varepsilon^{2}}\right\} v_{0}^{2}(0) \frac{1}{\sigma}(1+o(1)) .
\end{aligned}
$$

Для некоторых значений $p$ вычисления по формулам теоремы 1 можно провести точно. При $p=1$ и $p=2$ мы получаем следующие утверждения.

Пусть $\operatorname{Ai}(y)$ обозначает функцию Эйри [20; 10.4$],$ [21; гл. 11], [22], $\mathrm{Ai}^{\prime}(y)$ - ее производную, $a_{1}^{\prime} \approx 1.0188$ - первый нуль функции $\mathrm{Ai}^{\prime}(-y)$. 
СлЕДСтвиЕ 1 (малые уклонения в $L^{1}$-норме). При $\varepsilon \rightarrow 0$ выполнены соотноиения

$$
\mathrm{P}\left\{\int_{0}^{1}|w(t)| d t \leqslant \varepsilon\right\}=b \varepsilon \exp \left\{-\frac{b_{1}}{\varepsilon^{2}}\right\}(1+o(1)),
$$

где $b_{1}=\frac{2}{27}\left(a_{1}^{\prime}\right)^{3} \approx 0.07833$,

$$
\begin{gathered}
b=\frac{9}{2 \sqrt{2 \pi}\left(a_{1}^{\prime}\right)^{3 / 2}} M, \quad M=\frac{1}{3 a_{1}^{\prime} \operatorname{Ai}\left(-a_{1}^{\prime}\right)}\left(1+3 \int_{0}^{a_{1}^{\prime}} \operatorname{Ai}(-y) d y\right) \approx 1.48257 \\
\mathrm{P}\left\{\int_{0}^{1}\left|w_{0}(t)\right| d t \leqslant \varepsilon\right\}=\frac{1}{\sqrt{6 b_{1}}} \exp \left\{-\frac{b_{1}}{\varepsilon^{2}}\right\}(1+o(1)) .
\end{gathered}
$$

Заметим, что формулы (1.19), (1.21) можно получить, применяя классический метод Лапласа, из результатов статей [23], [24], [3; теорема 3.13].

СлеДСТвиЕ 2 (малые уклонения в $L^{2}$-норме). При $\varepsilon \rightarrow 0$ выполнены соотношения

$$
\begin{aligned}
& \mathrm{P}\left\{\int_{0}^{1} w^{2}(t) d t \leqslant \varepsilon^{2}\right\}=\frac{4}{\sqrt{\pi}} \varepsilon \exp \left\{-\frac{1}{8 \varepsilon^{2}}\right\}(1+o(1)) \\
& \mathrm{P}\left\{\int_{0}^{1} w_{0}^{2}(t) d t \leqslant \varepsilon^{2}\right\}=\sqrt{\frac{8}{\pi}} \exp \left\{-\frac{1}{8 \varepsilon^{2}}\right\}(1+o(1)) .
\end{aligned}
$$

Результаты следствия 2 были известны раннее (см., например, [1], [2], [3; §3]).

В качестве побочного результата в статье получено следующее утверждение, представляющее и самостоятельный интерес.

ПРЕДЛОЖЕНИЕ 1 (точная асимптотика типа Лапласа для времен пребывания). Для $p>0$ при $T \rightarrow \infty$ имеют место соотношения

$$
\begin{aligned}
& \mathrm{P}\left\{\int_{-\infty}^{\infty}|x|^{p} L_{T}(w, d x)<1\right\} \\
& =\frac{1}{\sqrt{T}} \exp \left\{-T b_{p}\right\} v_{0}(0) \int_{-\infty}^{\infty} v_{0}(x) d x \frac{1}{\sqrt{2 \pi} \sigma}(1+o(1)), \\
& \mathrm{P}\left\{\int_{-\infty}^{\infty}|x|^{p} L_{T}(w, d x)<1 \mid w(T)=0\right\}=\exp \left\{-T b_{p}\right\} v_{0}^{2}(0) \frac{1}{\sigma}(1+o(1)) .
\end{aligned}
$$

\section{$\S 2$. Связь задачи о малых уклонениях с теорией больших уклонений для времен пребывания}

Функционал времени пребывания случайного процесса, введенњый П. Леви [25], оказался очень эффективным и полезньм средством для изучения множества задач теории случайных процессов (см. [18], [25]-[28]). В частности, этот функционал и связанный с ним функционал локального времени играют важную роль при изучении асимптотических свойств распределений марковских процессов [9], [11], [29], 
[30], [31; гл. 6], ввиду того что для них построена теория больших уклонений. Дадим соответствуюшие определения и утверждения применительно к нашему случаю винеровского процесса, траектории которого, как известно, непрерывны почти наверное (п.н.).

Пусть $C[0, \infty)$ обозначает множество непрерывных функций, определенных на $[0, \infty)$ со значениями в $\mathbb{R}$. Для каждого $t>0, z \in C[0, \infty)$ и борелевского $B$ из $\mathbb{R}$ обозначим через

$$
L_{t}(z, B)=\frac{1}{t} \int_{0}^{t} I_{B}(z(s)) d s
$$

часть времени из $[0, t]$, которую фиксированная траектория $z=z(\cdot)$ проводит в множестве $B$, здесь $I_{B}(\cdot)$ - индикатор множества $B$. Функционал $L_{t}(z, B)$ кратко называют (нормированным) временем пребъвания.

Локальное время $l_{t}(z, y), y \in \mathbb{R}$, определяется как плотность времени пребывания (когда она существует):

$$
L_{t}(z, B)=\int_{B} l_{t}(z, y) d y
$$

Имеет место следуюшая важная

Теорема 2. Пусть действительная функиия $g$ измерима по Борелю и интегрируема на любом компактном множестве из $\mathbb{R}$. Тогда для каждого $t>0$ выполнено равенство

$$
\mathrm{P}\left\{\int_{-\infty}^{\infty} g(x) L_{t}(w, d x)=\frac{1}{t} \int_{0}^{t} g(w(s)) d s\right\}=1 .
$$

ДоКАЗАТЕЛЬСТво этого утверж дения имеется, например, в [26; гл. $3, \S 4]$ и $[28$; $\S 7.2]$, где определение локального времени (времени пребывания) отличается от наших (2.1) и (2.2) на некоторый фиксированный множитель.

Приведем еще один нужный нам результат.

ПРЕДЛОЖЕнИЕ 2 (масштабная инвариантность, или автомодельность, винеровского процесса). Пусть $\{w(s), s \geqslant 0\}-$ винеровский прочесс. Тогда для любого фиксированного $T>0$ прочесс

$$
\left\{T^{-1 / 2} w(T s), s \geqslant 0\right\}
$$

также является винеровским прочессом.

ДокАЗАТЕЛЬСтво этого факта имеется, например, в [17; § 2.1].

Лемма 1. Для любого фиксированного $T>0$

i) процессы $\left\{T^{1 / 2} w(s), s \geqslant 0\right\}$ u $\{w(T s), s \geqslant 0\}$ имеют одинаковое распределение;

ii) процессы $\left\{\left[T^{1 / 2} w(s) \mid w(1)=0\right], s \geqslant 0\right\} u\{[w(T s) \mid w(T)=0], s \geqslant 0\}$ имеют одинаковое распределение. 
ДокАЗАТЕЛЬСтво. Утверждение п. і) следует непосредственно из предложения 2. Утверждение іi) вытекает из п. i).

ЛЕмма 2. Пусть $p>0$. Тогда для любого фиксированного $T>0$ выполненьц равенства

$$
\begin{aligned}
T^{p / 2} \int_{0}^{1}|w(t)|^{p} d t & =\int_{-\infty}^{\infty}|x|^{p} L_{T}(w, d x) \\
T^{p / 2} \int_{0}^{1}\left|w_{0}(t)\right|^{p} d t & =\left\{\int_{-\infty}^{\infty}|x|^{p} L_{T}(w, d x) \mid w(T)=0\right\}
\end{aligned}
$$

ДокАЗАТЕЛЬСтво. Докажем (2.5). Согласно п. і) леммы 1 и предложению 2 имеем цепочку равенств

$$
T^{p / 2} \int_{0}^{1}|w(t)|^{p} d t=\int_{0}^{1}|w(T t)|^{p} d t=\frac{1}{T} \int_{0}^{T}|w(t)|^{p} d t=\int_{-\infty}^{\infty}|x|^{p} L_{T}(w, d x) \text { п.н. }
$$

Аналогично, с учетом того, что $w_{0}(t)=\{w(t) \mid w(1)=0\}$, доказывается (2.6). Лемма 2 доказана.

\section{§ 3. Метод Лапласа для малых уклонений. Доказательство теоремы 1}

Вывод утверждений типа теоремы 1 основан на свойстве автомодельности винеровского процесса, соотношениях типа (2.5) и результатах о точной асимптотике больших уклонений для времен пребывания винеровского процесса. Мы проведем подробно доказательство формулы (1.17) теоремы 1 и укажем схему вывода формулы (1.18).

В силу равенства (2.5) для любого фиксированного $T>0$ выполнено ключевое соотношение

$$
\mathrm{P}\left\{\int_{0}^{1}|w(t)|^{p} d t<\varepsilon^{p}\right\}=\mathrm{P}\left\{\int_{-\infty}^{\infty}|x|^{p} L_{T}(w, d x)<1\right\}, \quad \varepsilon=T^{-1 / 2} .
$$

Как видим, нужно найти точную асимптотику вероятности в правой части (3.1) при $T \rightarrow \infty$. Для этого мы используем теорию больших уклонений, развитую в работах [11], [13], [30].

Приведем некоторые результаты из [11], [30; III] для нашего случая винеровского процесса.

Обозначим через $\mathscr{M}$ метрическое пространство вероятностных мер на $\mathbb{R}$, снабженное метрикой Прохорова $\rho$ и борелевской $\sigma$-алгеброй. Везде ниже $\mathrm{E}$ означает усреднение по основной вероятности $\mathrm{P}$.

Обозначим также

$$
\mathscr{H}:=\{v \in \mathscr{D}: N(v)=1\} .
$$

Заметим, что если $v \in \mathscr{H}$, то функция $v^{2}$ является вероятностной плотностью на $\mathbb{R}$, кроме того, $\lim _{|x| \rightarrow \infty} v(x)=0$.

ТЕОРЕмА 3 (принцип больших уклонений) [11], [30; III]. 
i) Семейство вероятностных мер $\left[\mathrm{P}\left\{L_{T}(w, \cdot) \in(\cdot)\right\}\right]_{T>0}$ удовлетворяет в пространстве $(\mathscr{M}, \rho)$ при $T \rightarrow \infty$ принципу больших уклонений с функиионалом действия $I(\nu)$ и скоростью $T$, где

$$
I(\nu):= \begin{cases}-\langle\mathscr{A} v, v\rangle, & v \in \mathscr{H}, \nu(d x)=v^{2}(x) d x \\ +\infty & \text { в противном случае. }\end{cases}
$$

ii) Таким образом, для любого открытого в $\mathscr{M}$ множества $\mathscr{C}$ выполнены неравенства

$$
\begin{aligned}
-\inf _{\nu \in \mathscr{C}} I(\nu) & \leqslant \liminf _{T \rightarrow \infty} \frac{1}{T} \log \mathrm{P}\left\{L_{T}(w, \cdot) \in \mathscr{C}\right\} \\
& \leqslant \limsup _{T \rightarrow \infty} \frac{1}{T} \log \mathrm{P}\left\{L_{T}(w, \cdot) \in \overline{\mathscr{C}}\right\} \leqslant-\inf _{\nu \in \overline{\mathscr{C}}} I(\nu),
\end{aligned}
$$

где $\overline{\mathscr{C}}$ - замъкание множества $\mathscr{C}$.

iii) Для слабо непрерывного функционала $F$ на М имеет место равенство

$$
\lim _{T \rightarrow \infty} \frac{1}{T} \log \mathrm{E} \exp \left\{-T F\left(L_{T}(w, \cdot)\right)\right\}=-\inf _{\nu \in \mathscr{M}}(F(\nu)+I(\nu)) .
$$

Напомним, что функционал действия $I(\nu)$ является неотрицательным, полунепрерывным снизу и имеет компактные множества уровня (см. [6; $§ 2$, определение 2.1]). Используя интегрирование по частям, получаем

$$
-\langle\mathscr{A} v, v\rangle=\frac{1}{2} \int_{-\infty}^{\infty}\left(v^{\prime}(x)\right)^{2} d x \geqslant 0,
$$

таким образом, функционал $-\langle\mathscr{A} v, v\rangle$ является выпуклым вниз.

В работе [30; III] теорема 3 сформулирована и доказана для общего случая марковских процессов, включая и винеровский процесс. Как известно, переходная плотность винеровского процесса относительно меры Лебега имеет вид

$$
p(t, x, y)=\frac{1}{\sqrt{2 \pi t}} \exp \left\{-\frac{(x-y)^{2}}{2 t}\right\}, \quad t \in[0, \infty), \quad x, y \in \mathbb{R}
$$

(см., например, [17], [18], [25]-[27]).

ЗАмечание 1. Точная асимптотика интеграла из (3.3) фактически вычислена в работе [11] (см. теоремы 2.19 и 2.20). Дело в том, что в [11] рассмотрен случай марковских процессов, для которых переходные вероятности имеют плотность относительно некоторой вероятностной референтной меры на $\mathbb{R}$. Внимательный анализ доказательства результатов из [11] показывает, что эти результаты справедливы и в случае винеровского процесса, для которого референтная мера есть мера Лебега. Строгий вывод этого факта можно провести с помощью метода, использованного в [30; III], где также был осушествлен перенос асимптотической теоремы о больших уклонениях, доказанный первоначально для вероятностной референтной меры [30; I], на случай меры Лебега. 
Отметим, что в формуле (3.1) участвует линейный функционал

$$
F_{0}(\nu):=\int_{-\infty}^{\infty}|x|^{p} \nu(d x), \quad \nu \in \mathscr{M} .
$$

Введем также следующий квадратичный функционал, "ассоциированный" с функционалами $G$ из $(1.3)$ и $F_{0}$ :

$$
U(v):=\int_{-\infty}^{\infty}|x|^{p} v^{2}(x) d x \equiv\left\langle|x|^{p} v, v\right\rangle, \quad v \in \mathscr{D} .
$$

Полезна также иная запись функционала действия

$$
J(v):= \begin{cases}-\langle\mathscr{A} v, v\rangle, & v \in \mathscr{H} \\ +\infty, & v \in L^{2} \backslash \mathscr{H} .\end{cases}
$$

Легко видеть, что

$$
F_{0}(\nu) \equiv U(v), \quad I(\nu) \equiv J(v), \quad \nu(d x)=v^{2}(x) d x .
$$

В зависимости от ситуации нам будет удобно работать или в пространстве функций $L^{2}$, или в пространстве мер $\mathscr{M}$.

\section{1. Логарифмическая асимптотика.}

ЛЕмма 3. Для любого $р>0$ справедливы соотношения

$$
\begin{aligned}
\lim _{\varepsilon \rightarrow 0} & \varepsilon^{2} \log \mathrm{P}\left\{\int_{0}^{1}|w(t)|^{p} d t<\varepsilon^{p}\right\}=\lim _{\varepsilon \rightarrow 0} \varepsilon^{2} \log \mathrm{P}\left\{\int_{0}^{1}\left|w_{0}(t)\right|^{p} d t<\varepsilon^{p}\right\} \\
= & \lim _{T \rightarrow \infty} \frac{1}{T} \log \mathrm{P}\left\{\int_{-\infty}^{\infty}|x|^{p} L_{T}(w, d x)<1\right\} \\
= & -\inf \{J(v): v \in \mathscr{H}, v \geqslant 0, U(v) \leqslant 1\} .
\end{aligned}
$$

ДокАЗАТЕЛЬСтво. При $p \geqslant 1$ первые два равенства формулы (3.7) следуют из [4; лемма 2.3] и формулы (3.1). Покажем, как доказывается последнее равенство в (3.7). Для открытого в $\mathscr{M}$ множества $\mathscr{C}:=\left\{\nu \in \mathscr{M}: F_{0}(\nu)<1\right\}$ имеем

$$
\mathrm{P}\left\{\int_{-\infty}^{\infty}|x|^{p} L_{T}(w, d x)<1\right\}=\mathrm{P}\left\{F_{0}\left(L_{T}(w)\right)<1\right\}=\mathrm{P}\left\{L_{T}(w, \cdot) \in \mathscr{C}\right\} .
$$

Функционал $J(v)$ на своей эффективной области (см. [32; гл. 1, п. 2.1]) является гладким выпуклым функционалом, квадратические функционалы $N(v), U(v)$ дифференцируемы, поэтому

$$
\begin{aligned}
\inf _{\nu \in \mathscr{C}} I(\nu) & =\inf \{J(v): v \in \mathscr{H}, U(v)<1\}=\inf \{J(v): v \in \mathscr{H}, U(v) \leqslant 1\} \\
& =\inf _{\nu \in \overline{\mathscr{C}}} I(\nu),
\end{aligned}
$$

поскольку в рассматриваемой гладкой экстремальной задаче с ограничениями для функционала $J(v)$ строгое неравенство можно заменить на нестрогое (см. [33; гл. 3, п. 3.2.1], а также лемму 4 ниже).

Из формулы (3.9) в силу п. ii) теоремы 3 следует последнее равенство в (3.7). Условие $v \geqslant 0$ появляется вследствие инвариантности рассматриваемых функционалов относительно замены знака у аргумента на противоположный. Лемма 3 доказана. 
ЗАмечание 2. Первое равенство в (3.7) также можно доказать только на основе формулы (3.1) и принципа больших уклонений для времен пребывания броуновского моста. Можно доказать, что семейство распределений времен пребывания броуновского моста, т.е. семейство вероятностных мер (ср. п. іi) леммы 1 ) $\left[\mathrm{P}\left\{L_{T}(w, \cdot) \in(\cdot) \mid w(T)=0\right\}\right]_{T>0}$, удовлетворяет в пространстве $(\mathscr{M}, \rho)$ при $T \rightarrow \infty$ принципу больших уклонений с тем же самьм функционалом действия $I(\nu)$ и той же скоростью $T$.

Итак, согласно формуле (3.7) нужно решить следуюшую экстремальную задачу для неотрицательного квадратичного функционала:

$$
-\langle\mathscr{A} v, v\rangle \rightarrow \inf , \quad v \in \mathscr{H}, \quad v \geqslant 0, \quad U(v) \leqslant 1 .
$$

\section{2. Решение экстремальной задачи (3.10).}

Лемма 4. і) Экстремальная задача (3.10) имеет единственное решение функцию $v_{0}$, определенную в формуле (1.9). При этом выполнены равенства

$$
\left(\mathscr{A} v_{0}\right)(x)=-V(x) v_{0}(x), \quad x \in \mathbb{R}
$$

$u$

$$
\inf \{J(v): v \in \mathscr{H}, v \geqslant 0, U(v) \leqslant 1\}=-\left\langle\mathscr{A} v_{0}, v_{0}\right\rangle=\beta_{0}-\lambda_{0}=b_{p}>0,
$$

где постояннье $\lambda_{0}, \beta_{0}$ и $b_{p}$ определень в формулах (1.8), (1.16).

ii) Функция v $v_{0}$ есть единственное решение следующей граничной задачи для уравнения ІІрёдингера с симметричным потенциалом $|x|^{p}$ :

$$
\left\{\begin{array}{l}
\frac{1}{2} v^{\prime \prime}(x)-\lambda_{0}|x|^{p} v(x)=-\beta_{0} v(x), \quad x \in \mathbb{R} \\
v(-\infty)=v(\infty)=0 \\
\int_{-\infty}^{\infty} v^{2}(x) d x=1
\end{array}\right.
$$

причем

$$
\int_{-\infty}^{\infty}|x|^{p} v^{2}(x) d x=1
$$

ДоКАЗАТЕЛЬСтво. Применяя метод множителей Лагранжа [33; гл. 3, п. 3.2.1], найдем все локальные экстремумы задачи (3.10), затем выделим из них тот, который доставляет глобальный минимум в данной задаче. Функция Лагранжа задается формулой

$$
\mathscr{L}(v)=-\lambda_{1}\langle\mathscr{A} v, v\rangle+\lambda_{2}(U(v)-1)+\lambda_{3}(\langle v, v\rangle-1), \quad v \in \mathscr{D},
$$

где

$$
\lambda_{1} \geqslant 0, \quad \lambda_{2} \geqslant 0, \quad \lambda_{3} \in \mathbb{R}, \quad \lambda_{1}^{2}+\lambda_{2}^{2}+\lambda_{3}^{2}>0,
$$

- множители Лагранжа. Дифференцируя квадратичные функционалы, получаем, что для того чтобы точка $v$ доставляла минимум в задаче (3.10), необходимо выполнение условия стационарности

$$
\mathscr{L}^{\prime}(v) \equiv-2 \lambda_{1} \mathscr{A} v+2 \lambda_{2}|x|^{p} v+2 \lambda_{3} v=0, \quad v \in \mathscr{D},
$$


и условия дополняющей нежесткости

$$
\lambda_{2}(U(v)-1)=0, \quad \lambda_{3}(\langle v, v\rangle-1)=0 .
$$

Легко проверить, что в нашей задаче множитель $\lambda_{1}>0$. Действительно, предположим, что $\lambda_{1}=0$. Тогда соотношение (3.17) запишется в виде $2 v\left(\lambda_{2}|x|^{p}+\lambda_{3}\right)=0$, отсюда следует, что либо $v \equiv 0$ - это противоречит (3.14), либо $\lambda_{2}=\lambda_{3}=0$ - это противоречит последнему неравенству из (3.16). Итак, $\lambda_{1}>0$, обозначим $\lambda=\lambda_{2} / \lambda_{1}>0, \beta=-\lambda_{3} / \lambda_{1} \in \mathbb{R}$. Исследуя соотношения (3.15)-(3.18), несложно убедиться, что для функции $v$, доставляющей локальньй экстремум задачи (3.10), найдутся числа $\lambda=\lambda(v), \beta=\beta(v)$ такие, что тройка $(v, \lambda, \beta)$ будет удовлетворять следующей граничной задаче:

$$
\left\{\begin{array}{l}
\frac{1}{2} v^{\prime \prime}(x)-\lambda|x|^{p} v(x)=-\beta v(x), \quad x \in \mathbb{R} \\
v(-\infty)=v(\infty)=0 \\
\int_{-\infty}^{\infty} v^{2}(x) d x=1 \\
\int_{-\infty}^{\infty}|x|^{p} v^{2}(x) d x=1
\end{array}\right.
$$

Перейдем от (3.19) к более простой задаче. Сделаем замену переменных

$$
t=\lambda^{1 /(2+p)} x, \quad y(t)=\lambda^{-1 /(2(2+p))} v(x), \quad x \in \mathbb{R}
$$

Тогда произойдет “разделение параметров” и задача (3.19) запишется в виде

$$
\begin{aligned}
& \left\{\begin{array}{l}
-\frac{1}{2} y^{\prime \prime}(t)+|t|^{p} y(t)=\varkappa y(t), \quad t \in \mathbb{R} \\
y(-\infty)=y(\infty)=0 \\
\int_{-\infty}^{\infty} y^{2}(t) d t=1
\end{array}\right. \\
& \int_{-\infty}^{\infty}|t|^{p} y^{2}(t) d t=\lambda^{p /(2+p)}
\end{aligned}
$$

где

$$
\varkappa:=\frac{\beta}{\lambda^{2 /(2+p)}}
$$

- новый параметр. Согласно спектральной теории операторов Шрёдингера с растущим потенциалом (см. [16; гл. $9, \S 2$, п. 2]) задача $(3.21)$ имеет счетное число решений $\left(y_{n}, \varkappa_{n}\right), n=0,1,2, \ldots$, где

$$
0<\varkappa_{0}<\varkappa_{1}<\varkappa_{2}<\cdots<\varkappa_{n}<\cdots
$$

- собственные значения, $\left\{y_{n}\right\}$ - соответствующие им нормированные собственные функции оператора Шрёдингера $\mathscr{B}$, определенного в (1.7). 
Найдем число $\lambda_{n}$, которое определяется по формуле (3.22) с $y=y_{n}$. Для простоты записи индекс $n$ писать не будем. Умножим первое равенство в (3.21) на функцию $y(t)$ и проинтегрируем почленно полученное равенство по $t$ от $-\infty$ до $\infty$, в результате, учитьвая (3.22) и два других равенства из (3.21), получим

$$
-\frac{1}{2} \int_{-\infty}^{\infty} y^{\prime \prime}(t) y(t) d t=\varkappa-\lambda^{p /(2+p)} .
$$

Далее, запишем первое равенство в (3.21) в виде двух формул

$$
-\frac{1}{2} y^{\prime \prime}(t)+t^{p} y(t)=\varkappa y(t), \quad t \in[0, \infty),
$$

и

$$
-\frac{1}{2} y^{\prime \prime}(t)+(-t)^{p} y(t)=\varkappa y(t), \quad t \in(-\infty, 0] .
$$

Зафиксируем $s \geqslant 0$. Умножим равенство (3.25) на функцию $y^{\prime}(t)$ и проинтегрируем почленно полученное равенство по $t$ от $s$ до $\infty$, в результате получим

$$
-\frac{1}{2} \int_{s}^{\infty} y^{\prime \prime}(t) y^{\prime}(t) d t+\int_{s}^{\infty} t^{p} y(t) y^{\prime}(t) d t=\varkappa \int_{s}^{\infty} y(t) y^{\prime}(t) d t .
$$

Используя интегрирование по частям, находим

$$
\int_{s}^{\infty} t^{p} y(t) y^{\prime}(t) d t=\frac{1}{2} \int_{s}^{\infty} t^{p} d y^{2}(t)=-\frac{1}{2} s^{p} y^{2}(s)-\frac{p}{2} \int_{s}^{\infty} t^{p-1} y^{2}(t) d t .
$$

Подставляя это соотношение в (3.27) и выполняя несложный подсчет, убеж даемся, что из формулы (3.27) вытекает следуюшее равенство:

$$
\frac{1}{2}\left(y^{\prime}(s)\right)^{2}-s^{p} y^{2}(s)-p \int_{s}^{\infty} t^{p-1} y^{2}(t) d t=-\varkappa y^{2}(s), \quad s \geqslant 0 .
$$

Проинтегрируем почленно равенство (3.28) по $s$ от 0 до $\infty$, применяя теорему $Ф$ убини, в результате получим

$$
\frac{1}{2} \int_{0}^{\infty}\left(y^{\prime}(s)\right)^{2} d s=(p+1) \int_{0}^{\infty} s^{p} y^{2}(s) d s-\varkappa \int_{0}^{\infty} y^{2}(s) d s .
$$

Совершая аналогичные операции с равенством (3.26), приходим к формуле

$$
\frac{1}{2} \int_{-\infty}^{0}\left(y^{\prime}(s)\right)^{2} d s=(p+1) \int_{-\infty}^{0}(-s)^{p} y^{2}(s) d s-\varkappa \int_{-\infty}^{0} y^{2}(s) d s .
$$

Складывая почленно формулы (3.29), (3.30) и используя (3.21), (3.22), получаем

$$
\frac{1}{2} \int_{-\infty}^{\infty}\left(y^{\prime}(s)\right)^{2} d s=(p+1) \lambda^{p /(2+p)}-\varkappa .
$$

Из формул (3.24) и (3.31) окончательно находим

$$
\lambda_{n}=\left(\frac{2 \varkappa_{n}}{2+p}\right)^{(2+p) / p}, \quad n=0,1,2, \ldots
$$


Итак, согласно вышеизложенному с учетом формул (3.20), (3.23) заключаем, что задача (3.19) также имеет счетное число решений $\left(v_{n}, \lambda_{n}, \beta_{n}\right), n=0,1,2, \ldots$, где

$$
v_{n}(x)=\lambda_{n}^{1 /(2(2+p))} y_{n}\left(\lambda_{n}^{1 /(2+p)} x\right), \quad x \in \mathbb{R}
$$

$\lambda_{n}$ определено в $(3.32)$ и

$$
\beta_{n}=\frac{2+p}{2} \lambda_{n}=\left(\frac{2}{2+p}\right)^{2 / p} \varkappa_{n}^{(2+p) / p}
$$

Кроме того, используя (3.19), (3.32)-(3.34), легко находим

$$
-\left\langle\mathscr{A} v_{n}, v_{n}\right\rangle=\beta_{n}-\lambda_{n}=p 2^{2 / p}\left(\frac{\varkappa_{n}}{2+p}\right)^{(2+p) / p}, \quad n=0,1,2, \ldots
$$

Формула (3.35) показывает, что минимум в экстремальной задаче (3.10) достигается в единственной точке $v_{0}$. Легко видеть, что при $n=0$ формулы (3.32)-(3.35) совпадают с формулами (1.8), (1.9), (3.12). Соотношение (3.11) следует из (1.12) и первой формулы в (3.13). Сама задача (3.13) вместе с формулой (3.14) представляет собой задачу (3.19), записанную для основной минимизирующей тройки $\left(v_{0}, \lambda_{0}, \beta_{0}\right)$. Лемма 4 полностью доказана.

ЗАмЕчАнИЕ 3. Как правило, собственные функции $y_{n}$ являются знакопеременнымми при $n=1,2, \ldots$ (ср. с осцилляционной теоремой Штурма в случае конечного отрезка [34; ч. 2 , п. 9.2]).

Отметим также, что утверждения лемм 3 и 4 согласуются с результатом В. Ли (формулы (1.3) и (2.2) из [4]) о логарифмических асимптотиках.

Следуюшие рассуждения аналогичны использованным в [11; с. 533].

Пусть $C_{b}(\mathbb{R})$ - банахово пространство ограниченных непрерывных действительных функций на $\mathbb{R}$ с нормой супремума: $\|g\|_{C_{b}(\mathbb{R})}:=\sup _{x \in \mathbb{R}}|g(x)|$.

Выберем последовательности $\left\{\phi_{n}\right\}_{n=1}^{\infty} \subset C_{b}(\mathbb{R})$ и $\left\{a_{n}\right\}_{n=1}^{\infty} \subset(0, \infty)$ так, чтобы вьполнялись следующие три условия:

1) $\phi_{n} \neq 0, n=1,2, \ldots$, и $\left\|\phi_{n}\right\|_{C_{b}(\mathbb{R})} \rightarrow 0$ при $n \rightarrow \infty$;

2) $\left\{\left\|\phi_{n}\right\|^{-1} \phi_{n}\right\}_{n=1}^{\infty}$ есть полный ортонормированный базис в $\left(L^{2},\|\cdot\|\right)$;

3) $\sum_{n=1}^{\infty} a_{n}\left\|\phi_{n}\right\|^{-2}<\infty$.

Введем новую вероятностную меру на $\mathbb{R}$

$$
\nu_{0}(d x):=v_{0}^{2}(x) d x
$$

3.3. Доказательство формулы (1.17). Дальнейшие рассуждения в данном пункте относятся только к случаю винеровского процесса. 


\subsection{1. Выделение малой полуокрестности точки минимума.}

Лемма 5. Для любого $\delta>0$ найдется такое $c=c(\delta)>0$, ито при $T \rightarrow \infty$ имеет место равенство

$$
\mathrm{P}\left\{F_{0}\left(L_{T}(w)\right)<1\right\}=K(T)\left(1+O\left(e^{-T c}\right)\right),
$$

2дe

$$
K(T)=\mathrm{P}\left\{F_{0}\left(L_{T}(w)\right)<1, \sup _{n=1,2, \ldots}\left|\int_{-\infty}^{\infty} \phi_{n} d\left(L_{T}(w)-\nu_{0}\right)\right|<\delta\right\} .
$$

ДоКАЗАТЕЛЬСТВо леммы основано на леммах 3,4 , полунепрерывности снизу функционала действия $I(\nu)$ и проводится стандартно. Роль окрестности

$$
\left\{\omega: \sup _{n=1,2, \ldots}\left|\int_{-\infty}^{\infty} \phi_{n} d\left(L_{T}(w)-\nu_{0}\right)\right|<\delta\right\}
$$

в контексте теории больших уклонений времен пребывания марковских процессов выявлена и объяснена в статье $[11 ;$ с. 556$]$.

Отметим здесь далеко идушую аналогию между теорией больших уклонений времен пребывания марковских процессов [11], [30], [35] и теорией больших уклонений сумм независимых одинаково распределенных случайных банаховозначных элементов [12], [13], [30].

3.3.2. Использование экспоненциального преобразования типа Крамера. Следуя работе $[11 ;$ с. 545,556$]$ и учитывая $(3.11)$, введем следующее экспоненциальное преобразование типа Крамера. Для фиксированного $T>0$ определим вероятностную меру на основном вероятностном пространстве (ср. с [13; с. 527]):

$$
\begin{aligned}
\mathrm{Q}_{T}(d \omega):= & \exp \left\{T \int_{-\infty}^{\infty} V(x) L_{T}(w, d x)\right\} \mathrm{P}(d \omega) \\
& \times\left(\operatorname{E} \exp \left\{T \int_{-\infty}^{\infty} V(x) L_{T}(w, d x)\right\}\right)^{-1} .
\end{aligned}
$$

В результате замены меры (3.39) получаем для вероятности (3.38) равенство

$$
K(T)=K_{1}(T) K_{2}(T),
$$

где

$$
\begin{gathered}
K_{1}(T)=\mathrm{E} \exp \left\{T \int_{-\infty}^{\infty} V(x) L_{T}(w, d x)\right\} \\
K_{2}(T)=\int_{\mathscr{O}(T)} \exp \left\{-T \int_{-\infty}^{\infty} V(x) L_{T}(w, d x)\right\} \mathrm{Q}_{T}(d \omega) \\
\mathscr{O}(T) \equiv \mathscr{O}(T, \delta):=\left\{\omega: F_{0}\left(L_{T}(w)\right)<1, \sup _{n=1,2, \ldots}\left|\int_{-\infty}^{\infty} \phi_{n} d\left(L_{T}(w)-\nu_{0}\right)\right|<\delta\right\} .
\end{gathered}
$$

Найдем точные асимптотики интегралов $K_{1}(T)$ и $K_{2}(T)$. 
3.3.3. Точная асимптотика интеграла $K_{1}(T)$. Заметим, что

$$
K_{1}(T)=\mathrm{E} \exp \left\{T F_{1}\left(L_{T}(w, \cdot)\right)\right\}
$$

где

$$
F_{1}(\nu):=\int_{-\infty}^{\infty} V(x) \nu(d x), \quad \nu \in \mathscr{M},
$$

- линейньй функционал.

Приведем формулировку теоремы 2.20 [11; п. (1)] с учетом замечания 1 и того, что вторая производная по Фреше линейного функционала равна нулю.

ТЕОРема 4 (точная асимптотика типа Лапласа для линейного функционала) [11]. Пусть для линейного функционала $\Psi: \mathscr{M} \rightarrow \mathbb{R}$ әкстремальная задача

$$
-\Psi\left(u^{2} d x\right)-\langle\mathscr{A} u, u\rangle \rightarrow \inf , \quad u \in \mathscr{H},
$$

имеет единственное решение $u_{0}$. Тогда при $T \rightarrow \infty$ справедливо соотношение

$\mathrm{E} \exp \left\{T \Psi\left(L_{T}(w, \cdot)\right)\right\}=\exp \left\{T\left(\Psi\left(u_{0}^{2} d x\right)+\left\langle\mathscr{A} u_{0}, u_{0}\right\rangle\right)\right\} u_{0}(0) \int_{-\infty}^{\infty} u_{0}(x) d x(1+o(1))$

ЛЕмма 6. При $T \rightarrow \infty$ выполнено соотношение

$$
K_{1}(T)=v_{0}(0) \int_{-\infty}^{\infty} v_{0}(x) d x(1+o(1))
$$

ДокАЗАТЕЛЬСтво. Применим теорему 4 к интегралу $(3.44)$, положив $\Psi=F_{1}$. Для этого решим экстремальную задачу

$$
-\int_{-\infty}^{\infty} V(x) u^{2}(x) d x-\langle\mathscr{A} u, u\rangle \rightarrow \inf , \quad u \in \mathscr{H} .
$$

Используя метод множителей Лагранжа, убеждаемся, что эта задача имеет единственное решение $v_{0}$ - то же самое, что и основная экстремальная задача (3.10). При этом в силу (3.11) находим

$$
\inf _{u \in \mathscr{H}}\left(-\int_{-\infty}^{\infty} V(x) u^{2}(x) d x-\langle\mathscr{A} u, u\rangle\right)=-\int_{-\infty}^{\infty} V(x) v_{0}^{2}(x) d x-\left\langle\mathscr{A} v_{0}, v_{0}\right\rangle=0 .
$$

Применение теоремы 4 доказывает формулу (3.45). Лемма 6 доказана.

3.3.4. Точная асимптотика интеграла $K_{2}(T)$. Используя $(3.15),(3.21)$, последнее равенство в $(3.25)$ и (3.26), получаем

$$
K_{2}(T)=\exp \left\{T\left\langle\mathscr{A} v_{0}, v_{0}\right\rangle\right\} K_{3}(T)
$$

где

$$
K_{3}(T):=\int_{\mathscr{O}(T)} \exp \left\{-T \int_{-\infty}^{\infty} V(x) d\left(L_{T}(w)-\nu_{0}\right)\right\} d \mathrm{Q}_{T}
$$


В работе [11; с. 551] имеется формула Тейлора для разложения общего нелинейного функционала $F: \mathscr{M} \rightarrow \mathbb{R}$ в окрестности некоторой точки $m_{0} \in \mathscr{M}$. Применяя эту формулу для нашего линейного функционала $F_{0}$, получаем

$$
F_{0}\left(L_{T}(w)\right)=F_{0}\left(\nu_{0}\right)+\int_{-\infty}^{\infty} V(x) d\left(L_{T}(w)-\nu_{0}\right), \quad \omega \in \mathscr{O}_{T} .
$$

Используя (3.49) с учетом того, что $F_{0}\left(\nu_{0}\right)=1$, запишем интеграл (3.48) в виде

$$
K_{3}(T):=\int_{\mathscr{O}_{1}(T)} \exp \left\{-T \int_{-\infty}^{\infty} V(x) d\left(L_{T}(w)-\nu_{0}\right)\right\} d \mathrm{Q}_{T}
$$

где

$$
\begin{aligned}
\mathscr{O}_{1}(T):=\left\{\omega: \int_{-\infty}^{\infty} V(x) d\left(L_{T}(w)-\nu_{0}\right)<0,\right. \\
\left.\sup _{n=1,2, \ldots}\left|\int_{-\infty}^{\infty} \phi_{n} d\left(L_{T}(w)-\nu_{0}\right)\right|<\delta\right\} \equiv \mathscr{O}(T)
\end{aligned}
$$

- иная форма записи множества $\mathscr{O}(T)$.

Для нахождения асимптотики интеграла $K_{3}(T)$ применим следующую лемму.

ЛЕмма 7 (центральная предельная теорема). і) Случайная мера

$$
\sqrt{T}\left(L_{T}(w)-\nu_{0}\right)
$$

при $T \rightarrow \infty$ слабо сходится относительно меры $\mathrm{Q}_{T} \kappa$ гауссовской случайной мере $\gamma$ на $\mathbb{R}$ со средним нуль.

ii) Для любого $s \in \mathbb{R}$ выполнено соотношение

$$
\lim _{T \rightarrow \infty} \mathrm{Q}_{T}\left\{\omega: \sqrt{T} \int_{-\infty}^{\infty} V(x) d\left(L_{T}(w)-\nu_{0}\right)<s\right\}=\Phi\left(\frac{s}{\sigma}\right),
$$

где $\Phi$ - стандартная нормальная функиия распределения и $\sigma$ определено $\boldsymbol{\theta}(1.14)$.

ДоКАЗАТЕЛЬСТво. Утверждение і) леммы выводится с помощью леммы 2.12 из [11] и замечания 1 . Мы здесь докажем п. ii), т.е. покажем, как получается формула (1.14) для $\sigma^{2}$, отсутствуюшая в [11]. Обозначим через $L^{2}\left(d \nu_{0}\right)$ гильбертово пространство функций, заданных на $\mathbb{R}$, со скалярным произведением

$$
\langle y, z\rangle_{L^{2}\left(d \nu_{0}\right)}:=\int_{-\infty}^{\infty} y(x) z(x) v_{0}^{2}(x) d x
$$

Согласно п. i) леммы, определению меры $Q_{T}$ в $(3.39)$, виду характеристической функции меры $\gamma$ (см. [11; с. 557]) имеем

$$
\sigma^{2}=\int_{-\infty}^{\infty}[\mathscr{G} V](x) V(x) v_{0}^{2}(x) d x
$$


где, как следует из $[11 ;$ с. 545$]$, линейный оператор $\mathscr{G}$ отображает гильбертово пространство $L^{2}\left(d \nu_{0}\right)$ в себя по правилу

$$
\begin{aligned}
{\left[\mathscr{G}_{y}\right](x)=} & 2 \int_{0}^{\infty}\left(\frac{1}{v_{0}(x)} \exp \{t(\mathscr{A}+V)\}\left[v_{0} y\right](x)\right. \\
& \left.-\int_{-\infty}^{\infty} v_{0}(s) \exp \{t(\mathscr{A}+V)\}\left[v_{0} y\right](s) d s\right) d t .
\end{aligned}
$$

Несложно видеть, что в силу (3.11)

$$
[\mathscr{G} y](x)=\left[\mathscr{G}\left(y+c_{0}\right)\right](x), \quad y \in L^{2}\left(d \nu_{0}\right), \quad c_{0} \in \mathbb{R} .
$$

Используя (3.54), (3.55) и (1.10), получаем

$$
\begin{aligned}
{[\mathscr{G} V](x)=} & -\lambda_{0}[\mathscr{G} \psi](x)=-2 \lambda_{0} \int_{0}^{\infty}\left(\frac{1}{v_{0}(x)} \exp \{t(\mathscr{A}+V)\}\left[v_{0} \psi\right](x)\right. \\
& \left.-\int_{-\infty}^{\infty} v_{0}(s) \exp \{t(\mathscr{A}+V)\}\left[v_{0} \psi\right](s) d s\right) d t
\end{aligned}
$$

Поскольку $-V(x) \rightarrow \infty$ при $|x| \rightarrow \infty$, то самосопряженный оператор Шрёдингера $-\left.(\mathscr{A}+V)\right|_{E_{1}}: E_{1} \rightarrow E_{1}$ имеет дискретный однократный действительный спектр (см. [16; гл. $9, \S 2$, п. 2])

$$
\rho_{1}<\rho_{2}<\cdots<\rho_{n}<\cdots,
$$

кроме того, из доказательства леммы 6 следует, что $\rho_{1}>0$. Таким образом, с учетом того, что полугруппа винеровского процесса является феллеровской [36; приложение $6, \S 3]$, [37; гл. 10$]$ с $C_{0}$-условием, приходим к выводу, что в наших условиях применима известная формула теории полугрупп (см. [16; гл. $3, \S 3$, п. 5]), согласно которой из (3.56) и (1.15) получаем

$$
[\mathscr{G} V](x)=\frac{2 \lambda_{0}}{v_{0}(x)}(\mathscr{A}+V)^{-1}\left[v_{0} \psi\right](x) .
$$

Из формул (3.53), (3.57), (1.10) и (1.15) следует соотношение (1.14). Пункт ii) леммы 7 доказан.

Применение леммы 7 к интегралу (3.50) дает

$$
K_{3}(T):=K_{4}(T)(1+o(1)), \quad T \rightarrow \infty
$$

где

$$
K_{4}(T):=\int_{-\infty}^{0} e^{\sqrt{T} s} d \Phi\left(\frac{s}{\sigma}\right)
$$

Используя асимптотику

$$
1-\Phi(x)=\frac{1}{\sqrt{2 \pi} x} e^{-x^{2} / 2}(1+o(1)), \quad x \rightarrow \infty,
$$

находим

$$
K_{4}(T)=\frac{1}{\sqrt{2 \pi T} \sigma}(1+o(1)), \quad T \rightarrow \infty .
$$


Лемма 8. При $T \rightarrow \infty$ выполнено соотношение

$$
K_{2}(T)=\exp \left\{T\left\langle\mathscr{A} v_{0}, v_{0}\right\rangle\right\} \frac{1}{\sqrt{2 \pi T} \sigma}(1+o(1)) .
$$

ДокАЗАТЕльство. Утверждение леммы следует из (3.47), (3.58), (3.59).

3.3.5. Вывод формулы (1.17). Соотношение (1.17) теперь получаем из формул (3.1), (3.8), (3.37), (3.40), лемм 6 и 8.

Из формул (3.1) и (1.17) получаем первое утверждение предложения 1.

3.4. Доказательство формулы (1.18). Формула (1.18) доказывается по той же схеме, что и формула (1.17). Здесь мы укажем лишш на основные моменты, в частности, объясним разницу в поведении степенных членов в асимптотиках (1.17) и (1.18). Напомним, что точные асимптотики больших уклонений в $L^{p}$-норме для винеровского процесса и броуновского моста имеют одинаковые степенные члены (см. [38]).

Лемма 9. Пусть $p>0$. Тогда для любого фиксированного $T>0$ выполнено равенство

$$
\mathrm{P}\left\{\int_{0}^{1}\left|w_{0}(t)\right|^{p} d t<\varepsilon^{p}\right\}=\mathrm{P}\left\{\int_{-\infty}^{\infty}|x|^{p} L_{T}(w, d x)<1 \mid w(T)=0\right\}, \quad \varepsilon=T^{-1 / 2} .
$$

ДокАЗАТЕЛЬСтво. Утверждение леммы следует из формулы (2.6).

Аналог теоремы 4 для броуновского моста имеет следуюший вид.

TЕОРЕМА 5 (точная асимптотика для линейного функционала в случае броуновского моста) [11]. Пусть для линейного функиионала $\Psi: \mathscr{M} \rightarrow \mathbb{R}$ әкстремальная задача

$$
-\Psi\left(u^{2} d x\right)-\langle\mathscr{A} u, u\rangle \rightarrow \inf , \quad u \in \mathscr{H},
$$

имеет единственное решение $u_{0}$. Тогда при $T \rightarrow \infty$ справедливо соотношение

$$
\begin{aligned}
& \mathrm{E}\left[\exp \left\{T \Psi\left(L_{T}(w, \cdot)\right)\right\} \mid w(T)=0\right] \\
& \quad=\exp \left\{T\left(\Psi\left(u_{0}^{2} d x\right)+\left\langle\mathscr{A} u_{0}, u_{0}\right\rangle\right)\right\} u_{0}^{2}(0) \frac{1}{\sqrt{2 \pi T}}(1+o(1)) .
\end{aligned}
$$

Утверждение теоремы 5 можно получить из теоремы 2.19 [11], учитьвая замечание 1 и то, что переходная плотность винеровского процесса удовлетворяет соотношению $p(T, 0,0)=(2 \pi T)^{-1 / 2}, T>0$.

Дальнейшее доказательство формулы (1.18) почти дословно повторяет пп. 3.3.13.3.5 вывода формулы (1.17). Разница в асимптотиках из теорем 4 и 5 влечет за собой упомянутую разницу в поведении степенных членов в асимптотиках (1.17) и (1.18).

Формула (1.18), а вместе с ней и теорема 1 доказаны.

Из формул (3.60) и (1.18) получаем второе утверждение предложения 1. 


\section{§4. Доказательство следствий 1 и 2}

Формулы (1.17) и (1.18) даны в терминах функции $v_{0}$, поэтому нам удобнее работать с этой функцией. Отметим, что задача (3.13), (3.14) определяет искомую тройку $\left(v_{0}, \lambda_{0}, \beta_{0}\right)$ единственным образом. Имея целью дальнейшее вычисление постоянной $\sigma^{2}$ по формуле (1.14), мы будем решать именно задачу (3.13), (3.14), предполагая, что параметры $\lambda_{0}, \beta_{0}$ неизвестны. Вообше, заметим, что утверждения и формулы, связанные с функцией $v_{0}$, имеют более обшую природу, чем утверждения, в которых присутствует функция $y_{0}$.

Насколько известно автору, граничная задача (3.13), (3.14) имеет простое решение при $p=1$ и $p=2$.

4.1. Случай $p=1$. Доказательство следствия 1. На протяжении данного пункта $p=1$.

Пусть $\operatorname{Bi}(x)$ обозначает вторую функцию Эйри (см. [20;п. 10.4.1], [21; гл. 11, § 1]).

Лемма 10. Для функций Эйри выполнены следующие соотношения:

i) $\quad \mathrm{Ai}^{\prime \prime}(x)=x \operatorname{Ai}(x), \quad \mathrm{Bi}^{\prime \prime}(x)=x \operatorname{Bi}(x), \quad x \in \mathbb{R}$;

ii) $\operatorname{Ai}(x)=\exp \left\{-\frac{2}{3} x^{3 / 2}\right\} \frac{1}{2 \sqrt{\pi} x^{1 / 4}}(1+o(1)), \quad x \rightarrow \infty$,

$$
\operatorname{Bi}(x)=\exp \left\{\frac{2}{3} x^{3 / 2}\right\} \frac{1}{\sqrt{\pi} x^{1 / 4}}(1+o(1)), \quad x \rightarrow \infty ;
$$

iii) $\int \operatorname{Ai}^{2}(x) d x=x \operatorname{Ai}^{2}(x)-\left(\operatorname{Ai}^{\prime}(x)\right)^{2}, \quad x \in \mathbb{R}$,

$$
\int_{y}^{\infty} x \operatorname{Ai}^{2}(x) d x=\frac{1}{3}\left(-y^{2} \operatorname{Ai}^{2}(y)+y\left(\operatorname{Ai}^{\prime}(y)\right)^{2}-\mathrm{Ai}(y) \operatorname{Ai}^{\prime}(y)\right), \quad y \in \mathbb{R}
$$

Формулы пा. і) и іi) имеются в [20; пп. 10.4.1, 10.4.59, 10.4.63]. Соотношения (4.1), $(4.2)$ получаем из $[39 ;$ гл. 1, п. 1.8.2, (11), (14)].

ЛЕмма 11. Экстремаль $v_{0}$ является симметричной функиией и задается формулой

$$
\begin{aligned}
v_{0}(x) & =\frac{1}{\sqrt{3} \operatorname{Ai}\left(-a_{1}^{\prime}\right)} \operatorname{Ai}\left(\frac{2}{3} a_{1}^{\prime} x-a_{1}^{\prime}\right), & & x \geqslant 0, \\
v_{0}(-x) & =v_{0}(x), & x & \leqslant 0 .
\end{aligned}
$$

При этом

$$
\lambda_{0}=\frac{4}{27}\left(a_{1}^{\prime}\right)^{3}, \quad \beta_{0}=\frac{3}{2} \lambda_{0}=\frac{2}{9}\left(a_{1}^{\prime}\right)^{3}, \quad \varkappa_{0}=\frac{3}{2} \lambda_{0}^{1 / 3}=2^{1 / 3} a_{1}^{\prime} .
$$

ДокАЗАТЕЛЬСТво. При $p=1$ дифференциальное уравнение из формулы (3.13) принимает вид (индексы 0 опушены)

$$
v^{\prime \prime}(x)+(2 \beta-2 \lambda|x|) v(x)=0, \quad x \in \mathbb{R},
$$

условия связи из формул (3.13), (3.14) записьваются следующим образом:

$$
\int_{-\infty}^{\infty} v^{2}(x) d x=1, \quad \int_{-\infty}^{\infty}|x| v^{2}(x) d x=1 .
$$


Легко видеть, что если функция $v_{0}(x)$ есть решение уравнения (4.5) для значений $x \geqslant 0$, то функция $v_{0}(-x)$ удовлетворяет уравнению (4.5) для значений $x \leqslant 0$. Поэтому решим вначале следуюшее дифференциальное уравнение:

$$
v^{\prime \prime}(x)+(2 \beta-2 \lambda x) v(x)=0, \quad x \in[0, \infty) .
$$

Обозначим

$$
C:=(2 \lambda)^{1 / 3}>0, \quad a:=\frac{2 \beta}{C^{2}}=\frac{2 \beta}{(2 \lambda)^{2 / 3}}>0 .
$$

Сделаем в (4.7) замену переменных $t=C x \in[0, \infty), z(t)=v(x)$. Тогда уравнение (4.7) примет вид

$$
z^{\prime \prime}(t)-(t-a) z(t)=0, \quad t \in[0, \infty) .
$$

Положив $s=t-a, u(s)=z(t)$, запишем уравнение (4.9) в виде

$$
u^{\prime \prime}(s)-s u(s)=0, \quad s \in[-a, \infty) .
$$

Это - хорошо изученное дифференциальное уравнение, решениями которого являются функции Эйри $\operatorname{Ai}(s)$ и $\operatorname{Bi}(s)$ (см. [20; п. 10.4.1], [21; гл. 11, §1]). Нужное нам положительное решение, удовлетворяющее в силу (4.6) условию $u(\infty)=0$, представляет собой функцию Эйри $u(s)=\mathrm{Ai}(s), s \geqslant-a$, при этом должно выполняться неравенство $a \leqslant a_{1}$, где $a_{1} \approx 2.3381$ - первый нуль функции Эйри $\mathrm{Ai}(-y)$. Итак, согласно вьшеизложенному дифференциальное уравнение (4.7) имеет единственное положительное решение

$$
v_{0}(x)=d \operatorname{Ai}(C x-a), \quad x \geqslant 0,
$$

где числа $d>0, C>0, a>0($ см. (4.9)) нужно определить, используя равенства (4.6) и условие дважды дифференцируемости экстремали $v_{0}$ в нуле. Положим

$$
v_{0}(x)=v_{0}(-x)=d \operatorname{Ai}(-C x-a), \quad x \leqslant 0 .
$$

Тогда, очевидно, $v_{0}^{\prime}(x)=-v_{0}^{\prime}(-x), x \in \mathbb{R}$, и, следовательно, $v_{0}^{\prime}(0+)=v_{0}^{\prime}(0-)=0$. Отсюда в силу (4.11), (4.12) находим $\mathrm{Ai}^{\prime}(-a)=0$ и $a=a_{1}^{\prime}$, поскольку $a \leqslant a_{1}$.

Условия связи (4.6) для функции (4.11), (4.12) принимают вид

$$
\int_{-a_{1}^{\prime}}^{\infty} \mathrm{Ai}^{2}(t) d t=\frac{C}{2 d^{2}}, \quad \int_{-a_{1}^{\prime}}^{\infty}\left(t+a_{1}^{\prime}\right) \mathrm{Ai}^{2}(t) d t=\frac{C^{2}}{2 d^{2}}
$$

Используя лемму 10, iii) и формулы (4.13), находим

$$
d=\frac{1}{\sqrt{3} \operatorname{Ai}\left(-a_{1}^{\prime}\right)}, \quad C=\frac{2}{3} a_{1}^{\prime},
$$

отсюда с учетом $(4.8),(4.11)$ и (1.8) получаем формулы (4.3) и (4.4). Лемма 11 доказана.

Нам понадобятся дальнейшие свойства функции Эйри. 
Лемма 12. Для функций Эйри выполнены следующие соотношения:

$$
\begin{gathered}
\left(\frac{\mathrm{Bi}(x)}{\mathrm{Ai}(x)}\right)^{\prime}=\frac{1}{\pi \mathrm{Ai}^{2}(x)}, \quad x \in \mathbb{R} . \\
\left(\frac{\mathrm{Ai}^{\prime}(x)}{\operatorname{Ai}(x)}\right)^{\prime}=x-\frac{\left(\mathrm{Ai}^{\prime}(x)\right)^{2}}{\operatorname{Ai}^{2}(x)}, \quad x \in \mathbb{R} . \\
\int \frac{\left(\mathrm{Ai}^{\prime}(x)\right)^{2}}{\operatorname{Ai}^{2}(x)} d x=\frac{x^{2}}{2}-\frac{\mathrm{Ai}^{\prime}(x)}{\operatorname{Ai}(x)}, \quad x \in \mathbb{R} . \\
\int x \frac{\left(\mathrm{Ai}^{\prime}(x)\right)^{2}}{\mathrm{Ai}^{2}(x)} d x=\frac{x^{3}}{3}-x \frac{\operatorname{Ai}^{\prime}(x)}{\operatorname{Ai}(x)}+\log \operatorname{Ai}(x), \quad x \in \mathbb{R} . \\
\int_{-a_{1}^{\prime}}^{\infty} x \operatorname{Ai}(x) \operatorname{Ai}^{\prime}(x) d x=0 .
\end{gathered}
$$

ДокАЗАТЕЛЬСТво. Формула (4.15) доказана с помощью вронскиана, равного $\mathscr{W}(\operatorname{Ai}(x), \operatorname{Bi}(x))=1 / \pi$, в [21; гл. 11, $\S 2$ п. 2.2]. Равенство (4.16) доказывается непосредственньм вычислением с учетом тождества $\mathrm{Ai}^{\prime \prime}(x)=x \mathrm{Ai}(x)$. Соотношения (4.17) и (4.18) получаются при помощи тождества (4.16) и интегрирования по частям. Формула (4.19) доказывается с использованием интегрирования по частям с учетом того, что функция $\mathrm{Ai}^{\prime}(x)$ монотонно убывает при $x \in[-a, 0]$ и монотонно возрастает при $x \in[0, \infty)$. Лемма 12 доказана.

Пусть $\psi(x)=|x|-1, x \in \mathbb{R}$, найдем

$$
g_{0}(x):=(\mathscr{A}+V)^{-1}\left[v_{0} \psi\right](x)
$$

- такую функцию, что

$$
\int_{-\infty}^{\infty} g_{0}(x) v_{0}(x) d x=0
$$

Лемма 13. Функиия $g_{0}(x)$ задается формулой

$$
\begin{gathered}
g_{0}(x)=\frac{9}{8\left(a_{1}^{\prime}\right)^{3}} v_{0}(x)+\frac{3 d}{2\left(a_{1}^{\prime}\right)^{2}} x \mathrm{Ai}^{\prime}\left(\frac{2}{3} a_{1}^{\prime} x-a_{1}^{\prime}\right), \quad x \geqslant 0, \\
g_{0}(-x)=g_{0}(x), \quad x \leqslant 0,
\end{gathered}
$$

где d определено в (4.14).

ДокАЗАТЕЛЬСтво. В силу определения (4.20) функция $g_{0}$ удовлетворяет уравнению $(\mathscr{A}+V) g=v_{0} \psi$, или

$$
g^{\prime \prime}(x)+\left(2 \beta_{0}-2 \lambda_{0}|x|\right) g(x)=2 v_{0}(x)(|x|-1), \quad x \in \mathbb{R} .
$$

С учетом (4.3) легко видеть, что дифференциальное уравнение (4.23) имеет четное решение $g(x)=g(-x), x \in \mathbb{R}$. Поэтому решим вначале следуюшее дифференциальное уравнение

$$
g^{\prime \prime}(x)+\left(2 \beta_{0}-2 \lambda_{0} x\right) g(x)=2 v_{0}(x)(x-1), \quad x \in[0, \infty) .
$$


Дифференциальное уравнение (4.24) является неоднородным по отношению к однородному уравнению (4.7). Зная два линейно независимых решения однородного уравнения (4.7), найдем, применяя известный метод [34; ч. $1, \S 24.2$, п. (б)], нужное нам решение уравнения (4.24), удовлетворяюшее условию (4.21).

В процессе доказательства леммы 11 мы установили, что однородное уравнение (4.7) имеет два линейно независимых решения $v_{0}(x)=d \mathrm{Ai}(C x-a)$ и $v_{1}(x)=$ $\operatorname{Bi}(C x-a), x \in[0, \infty)$. Здесь и ниже для краткости вновь обозначили $a=a_{1}^{\prime}$, $C=2 a_{1}^{\prime} / 3$. Следовательно, используя тождество (4.15) и [34; ч. $\left.1, \S 24.2\right]$, убеждаемся, что общее решение уравнения (4.24) имеет вид

$$
g(x)=C_{1} v_{0}(x)+C_{2} v_{1}(x)+2 v_{0}(x) \int \frac{1}{v_{0}^{2}(x)}\left(\int v_{0}^{2}(x)(x-1) d x\right) d x, \quad x \geqslant 0,
$$

где $C_{1}$ и $C_{2}$ - действительные константы. Из условия (4.21) и леммы $\left.10, \mathrm{ii}\right)$ следует, что в (4.25) $C_{2}=0$.

Используя формулы (4.1) и (4.2), находим

$$
\begin{aligned}
\int v_{0}^{2}(x)(x-1) d x= & \frac{d^{2}}{3 C^{2}}\left(C x(C x-a) \mathrm{Ai}^{2}(C x-a)-C x\left(\mathrm{Ai}^{\prime}(C x-a)\right)^{2}\right. \\
& \left.+\operatorname{Ai}(C x-a) \operatorname{Ai}^{\prime}(C x-a)\right), \quad x \geqslant 0 .
\end{aligned}
$$

Отсюда при помощи соотношений (4.17), (4.18) получаем

$$
\int \frac{1}{v_{0}^{2}(x)}\left(\int v_{0}^{2}(x)(x-1) d x\right) d x=\frac{1}{3 C^{2}} x \frac{\operatorname{Ai}^{\prime}(C x-a)}{\operatorname{Ai}(C x-a)}, \quad x \geqslant 0 .
$$

Из формул (4.25), (4.27) следует, что нужное нам решение уравнения (4.24) имеет вид

$$
g_{0}(x)=C_{1} v_{0}(x)+\frac{2 d}{3 C^{2}} x \mathrm{Ai}^{\prime}(C x-a), \quad x \geqslant 0 .
$$

Положим

$$
g_{0}(x)=g_{0}(-x), \quad x \leqslant 0 .
$$

Поскольку $v_{0}^{\prime}(0+)=v_{0}^{\prime}(0-)=0$, то несложно проверить, что $g_{0}^{\prime}(0+)=g_{0}^{\prime}(0-)=0$. Таким образом, учитывая условие (4.21), убеждаемся, что функция (4.28), (4.29) и есть единственно возможное в наших условиях решение уравнения (4.23).

Найдем постоянную $C_{1}$. Имеем согласно (4.6), (4.19), (4.23), (4.28), (4.29)

$$
0=\int_{-\infty}^{\infty} g_{0}(x) v_{0}(x) d x=2 \int_{0}^{\infty} g_{0}(x) v_{0}(x) d x=C_{1}-\frac{2 a}{9 C^{4}} .
$$

Отсюда с учетом (4.14) получаем

$$
C_{1}=\frac{9\left(a_{1}^{\prime}\right)^{-3}}{8}
$$

Из соотношений (4.28)-(4.30) следует формула (4.22). Лемма 13 доказана. 
ЛЕмма 14. Для функиий Эйри справедливы следующие соотношения:

$$
\begin{aligned}
\int_{0}^{\infty} \operatorname{Ai}(x) d x & =\frac{1}{3}, \\
\int_{-a_{1}^{\prime}}^{\infty} x^{2} \operatorname{Ai}(x) \operatorname{Ai}^{\prime}(x) d x & =-\frac{\left(a_{1}^{\prime}\right)^{2}}{6} \operatorname{Ai}^{2}\left(-a_{1}^{\prime}\right) .
\end{aligned}
$$

Равенство (4.31) следует из [39; гл. 2, п. 2.11.2, (1)]. Соотношение (4.32) вытекает из [39; гл. 1, п. 1.8.2, (18)].

Значения констант из формул (1.17), (1.18) приведены в следуюшей лемме.

Лемма 15. Имеют место соотношения

$$
\begin{gathered}
b_{1}=\beta_{0}-\lambda_{0}=\frac{2}{27}\left(a_{1}^{\prime}\right)^{3} \\
v_{0}(0)=\frac{1}{\sqrt{3}}, \quad \int_{-\infty}^{\infty} v_{0}(x) d x=\frac{1}{\sqrt{3} a_{1}^{\prime} \operatorname{Ai}\left(-a_{1}^{\prime}\right)}\left(1+3 \int_{0}^{a_{1}^{\prime}} \operatorname{Ai}(-x) d x\right) \\
\sigma^{2}=-4 \lambda_{0}^{2} \int_{0}^{\infty} x g_{0}(x) v_{0}(x) d x=\frac{4}{81}\left(a_{1}^{\prime}\right)^{3}
\end{gathered}
$$

ДоказАтЕльство. Соотношения (4.33) получаем из формул (1.16) и (4.4). Равенства (4.34) выводятся с помощью формул (4.3) и (4.31). Формула (4.35) доказывается с использованием соотношений $(1.14),(4.6),(4.20)-(4.22),(4.32)$. Лемма 15 доказана.

ДокАЗАТЕЛЬСтво слЕДСТвия 1. Формулы (1.19)-(1.21) получаем из соотношений $(1.17),(1.18)$ теоремы 1 с учетом леммы 15. Приближенные значения констант из следствия 1 найдены на основе таблиц 10.11-10.13 справочника [20;гл. 10]. Следствие 1 доказано.

4.2. Случай $p=2$. Доказательство следствия 2. На протяжении данного пункта $p=2$.

При $p=2$ дифференциальное уравнение из формулы (3.13) преврашается в хорошо изученное уравнение Вебера (см. (4.44) ниже и [34; ч. 3, гл. 2, п. 2.87], [20; гл. 19]). Решениями этого уравнения являются функции параболического цилиндра. Далее можно продолжать исследование по той же схеме, что и вьше. Отметим, однако, что экстремаль $v_{0}$ в данном случае вычисляется значительно проше, как показывает следующая ниже лемма.

Обозначим через

$$
\varphi(x):=\frac{1}{\sqrt{2 \pi}} \exp \left\{-\frac{x^{2}}{2}\right\}, \quad x \in \mathbb{R}
$$

стандартную гауссовскую плотность. Легко подсчитать, что

$$
\varphi^{\prime}(x)=-x \varphi(x), \quad x \in \mathbb{R}
$$


ЛЕмма 16. Экстремаль $v_{0}$ является симметричной функиией и задается формулой

$$
v_{0}(x)=\sqrt{\varphi(x)}=\frac{1}{(2 \pi)^{1 / 4}} \exp \left\{-\frac{x^{2}}{4}\right\}, \quad x \in \mathbb{R} .
$$

При этом

$$
\begin{gathered}
b_{2}=\beta_{0}-\lambda_{0}=\frac{1}{8}, \\
\lambda_{0}=\frac{1}{8}, \quad \beta_{0}=\frac{1}{4}, \quad \varkappa_{0}=\frac{1}{\sqrt{2}} .
\end{gathered}
$$

ДоказАтельство. Решим экстремальную задачу (3.10) с помошью неравенства Коши-Буняковского. Интегрируя по частям и применяя указанное неравенство, имеем для любой функции $v \in \mathscr{H}, U(v) \leqslant 1$ (см. (1.6), (3.5)),

$$
\begin{aligned}
1 & =\int_{-\infty}^{\infty} v^{2}(x) d x=2 \int_{-\infty}^{\infty}(-x v(x)) v^{\prime}(x) d x \\
& \leqslant 2\left(\int_{-\infty}^{\infty} x^{2} v^{2}(x) d x\right)^{1 / 2}\left(\int_{-\infty}^{\infty}\left(v^{\prime}(x)\right)^{2} d x\right)^{1 / 2} \leqslant 2\left(\int_{-\infty}^{\infty}\left(v^{\prime}(x)\right)^{2} d x\right)^{1 / 2} .
\end{aligned}
$$

Отсюда согласно (1.13) получаем неравенство

$$
-\langle\mathscr{A} v, v\rangle=\frac{1}{2} \int_{-\infty}^{\infty}\left(v^{\prime}(x)\right)^{2} d x \geqslant \frac{1}{8}, \quad v \in \mathscr{H}, \quad U(v) \leqslant 1 .
$$

Знаки равенства в (4.41) и (4.42) достигаются только в случае, когда

$$
-x v(x)=c_{0} v^{\prime}(x), \quad x \in \mathbb{R}, \quad U(v)=1,
$$

где $c_{0}$ - действительная константа. Решая дифференциальное уравнение (4.43) и учитывая условия $N(v)=1, U(v)=1$, получаем формулу $(4.38)$, при этом $c_{0}=2$.

Таким образом, $-\left\langle\mathscr{A} v_{0}, v_{0}\right\rangle=\frac{1}{8}$. Значения параметров $\lambda_{0}$ и $\beta_{0}$ находим, подставляя функцию $(4.38)$ в формулы $(3.13),(3.14)$ и производя несложные вычисления; в частности, при $p=2$ дифференциальное уравнение из формулы (3.13) принимает вид

$$
v^{\prime \prime}(x)+\left(2 \beta_{0}-2 \lambda_{0} x^{2}\right) v(x)=0, \quad x \in \mathbb{R} .
$$

Так мы получаем формулы (4.39) и (4.40). Лемма 16 доказана.

Пусть теперь $\psi(x)=x^{2}-1, x \in \mathbb{R}$, найдем

$$
g_{0}(x):=(\mathscr{A}+V)^{-1}\left[v_{0} \psi\right](x)
$$

- такую функцию, что

$$
\int_{-\infty}^{\infty} g_{0}(x) v_{0}(x) d x=0
$$

а также вычислим остальные константы из теоремы 1. 
ЛЕмма 17. і) Функиия $g_{0}(x)$ задается формулой

$$
g_{0}(x)=-\left(x^{2}-1\right) v_{0}(x), \quad x \in \mathbb{R} .
$$

ii) Справедливь следующие соотношения:

$$
v_{0}(0)=\frac{1}{(2 \pi)^{1 / 4}}, \quad \int_{-\infty}^{\infty} v_{0}(x) d x=\sqrt{2}(2 \pi)^{1 / 4}, \quad \sigma^{2}=\frac{1}{16} .
$$

ДокАЗАТЕльство. Утверждение і) леммы доказывается по той же схеме, что и лемма 13, только вычисления здесь намного проще. Вид функции (4.45) можно найти, используя (4.40) и [34; ч. 3, гл. 2, п. 2.87], где нужно положить $n=2$. Формулы (4.46) легко следуют из соотношений (1.14), (4.38), (4.40) и (4.45). Лемма 17 доказана.

ДокАЗАТЕЛЬСтво слЕДСТвИя 2. Формулы (1.22), (1.23) получаем из соотношений $(1.17),(1.18)$ теоремы 1 с учетом лемм 16 и 17. Следствие 2 доказано.

Вычисления по формулам теоремы 1 вполне возможны и для других значений $p$. Автор выражает благодарность рецензенту за полезные замечания.

\section{Список литературы}

1. Сытая Г.Н. О некоторых асимптотических представлениях для гауссовской меры в гильбертовом пространстве // Теория случ. процессов. 1974. Т. 2. С. 93-104.

2. Лифиии М. А. Гауссовские случайные функции. Киев: ТВ и МС, 1995.

3. Фаталов В. Р. Константы в асимптотиках вероятностей малых уклонений для гауссовских процессов и полей // УМН. 2003. Т. 58. № 4. С. 89-134.

4. $L i W . V$. Small ball probabilities for Gaussian Markov processes under the $L^{p}$-norm // Stochastic Process. Appl. 2001. V. 92. № 1. P. 87-102.

5. Lifshits M.A., Simon T. Small deviations for fractional stable processes // Preprint. № 177. Évry: Univ. d'Évry Prépubl., 2003.

6. Питербарг В. И., Фаталов В. Р. Метод Лапласа для вероятностных мер в банаховых пространствах // УМН. 1995. Т. 50. №6. С. 57-150.

7. Dembo A., Zeitouni $O$. Large deviations techniques and applications. New York: Springer-Verlag, 1998.

8. Фаталов В.Р. Асимптотики вероятностей малых уклонений в $L^{2}$-норме для двух классов гауссовских стационарных процессов // Теория вероятностей и ее прим. (в печати).

9. Donsker M.D., Varadhan S.R.S. On laws of the iterated logarithm for local times // Comm. Pure Appl. Math. 1977. V. 30. P. 707-753.

10. Боровков А. А., Могульский A.A. О вероятностях малых уклонений для случайных процессов // Труды Института математики СО АН СССР. Т. 13. Новосибирск: Наука, 1989. C. $147-168$.

11. Kusuoka S., Tamura Y. Precise estimate for large deviation of Donsker-Varadhan type // J. Fac. Sci. Univ. Tokyo, Sect. IA, Math. 1991. V. 38. P. 533-565.

12. Bolthausen E. Laplace approximations for sums of independent random vectors. I, II // Probab. Theory Related Fields. 1986. V. 72. № 2. P. 305-318; 1987. V. 76. № 2. P. 167-206.

13. Fatalov V.R. The Laplace method for computing exact asymptotics of distributions of integral statistics // Math. Methods Statist. 1999. V. 8. №4. P. 510-535.

14. Фаталов В. Р. Большие уклонения гауссовских мер в пространствах $l^{p}$ и $L^{p}, p \geqslant 2 / /$ Теория вероятностей и ее прим. 1996. Т. 41. № 3. С. 682-689.

15. Кач M. Несколько вероятностных задач физики и математики. М.: Наука, 1967.

16. Крейн С. Г. (ред.) Функциональньй анализ. М.: Наука, 1972.

17. Хида Т. Броуновское движение. М.: Наука, 1987. 
18. Бородин А.Н., Салминен П. Справочник по броуновскому движению. С.-Пб.: Лань, 2000 .

19. Канторович Л. В., Акилов Г. П. Функциональньй анализ. М.: Наука, 1977.

20. Абрамович М., Стиган И. (ред.) Справочник по специальньм функциям. М.: Наука, 1979.

21. Олвер Ф. Асимптотики и специальные функции. М.: Наука, 1990.

22. Яковлева Г. Д. Таблицы функций Эйри и их производных. М.: Наука, 1969.

23. Rice $S$. $O$. The integral of the absolute value of the pinned Wiener process - calculation of its probability density by numerical integration // Ann. Probab. 1982. V. 10. № 1. P. 240-243.

24. Takács L. On the distribution of the integral of the absolute value of the Brownian motion // Ann. Appl. Probab. 1993. V. 3. P. 186-197.

25. Леви П. Стохастические процессы и броуновское движение. М.: Наука, 1972.

26. Икәда Н., Ватанабә С. Стохастические дифференциальные уравнения и диффузионные процессы. М.: Наука, 1986.

27. Revuz D., Yor M. Continuous martingales and Brownian motion. Berlin: Springer-Verlag, 1999.

28. Чжун K., Уильямс P. Введение в стохастическое интегрирование. М.: Мир, 1987.

29. Donsker M.D., Varadhan S. R. S. Asymptotic evaluation of certain Wiener integrals for large time // Functional integration and its applications / ed. A.M. Arthur. Oxford: Oxford Univ. Press, 1975. P. 15-33.

30. Donsker M.D., Varadhan S.R.S. Asymptotic evaluation of certain Markov process expectations for large time. I, II, III, IV // Comm. Pure Appl. Math. 1975. V. 28. P. 1-47; 1975. V. 28. P. 279-301; 1976. V. 29. P. 389-461; 1983. V. 36. P. 525-565.

31. Simon B. Functional integration and quantum physics. New York: Academic Press, 1979.

32. Экланд И., Темам Р. Выпуклый анализ и вариационные проблемы. М.: Мир, 1979.

33. Алексеев B. М., Тихомиров В. М., Фомин С. В. Оптимальное управление. М.: Наука, 1979.

34. Камке Э. Справочник по обыпновенным дифференциальным уравнениям. М.: Наука, 1961.

35. Bolthausen E., Deuschel J.-D., Tamura Y. Laplace approximations for large deviations of nonreversible Markov processes. The nondegenerate case // Ann. Probab. 1995. V. 23. № 1. P. 236-267.

36. Булинский A. В., Ширяев A. Н. Теория случайных процессов. М.: Физматлит, 2003.

37. Вентцель А. Д. Курс теории случайных процессов. М.: Наука-Физматлит, 1996.

38. Фаталов В.Р. Асимптотики больших уклонений гауссовских процессов типа винеровского для $L^{p}$-функционалов, $p>0$, и гипергеометрическая функция // Матем. сб. 2003. T. 194. № 3. C. $61-82$.

39. Прудников А.П., Брычков Ю.А., Маричев О. И. Интегралы и ряды. Дополнительные главы. М.: Наука, 1986.

Московский государственнњй университет

Поступила в редакцию им. М.В. Ломоносова

05.09 .2003 и 24.08 .2004 